\title{
Kohlröschen - mehr als nur Nigritella nigra
}

\author{
Kurt Baumann
}

\begin{abstract}
Vanilla Orchid (Nigritella) is a common orchid genus in the Alps. For a long period botanists knew only the two species Red and Black Vanilla Orchid (Nigritella rubra and N. nigra). Today 18 species, subspecies and varieties are known. As Gymnadenia is a relative of Nigritella, some botanists united both genera (Nigritella included in Gymnadenia). Morphology, biology and areals of Nigritella are described.
\end{abstract}

\section{Zusammenfassung}

Das Kohlröschen (Nigritella) ist eine in den Alpen weit verbreitete Orchideen-Gattung. Lange unterschied man nur zwischen dem Roten und dem Schwarzen Kohlröschen (Nigritella rubra und N. nigra). Heute unterscheidet man 18 Arten, Unterarten und Varietäten. Gymnadenia ist eine mit Nigritella eng verwandte Gattung, weshalb Kohlröschen von manchen Botanikern auch als Gymnadenia bezeichnet werden. Äußeres Erscheinungsbild, Biologie und Verbreitung von Kohlröschen werden vorgestellt.

\section{Einführung}

Kohlröschen, was ist das oder was sind dies? Sicherlich ist damit weder die liebkosende Bezeichnung eines französischen Liebhabers für seine deutsche Geliebte (obwohl das sicher hübscher wäre als „Krautliesl“) noch der freudige Ausruf eines Gemüsebauers beim Anblick eines stattlichen Rosenkohls gemeint. Kohlröschen ist die Orchideen-Gattung Nigritella, zu der auch die Orchidee des Jahres 2007 gehört.

Der deutsche Name Kohlröschen, der in der neueren Literatur überwiegend benutzt wird (BAUMAnn 2005), bezieht sich wie auch ältere Bezeichnungen (Brändlein, Braunelle, Schwärzlein, Möhrli) auf die schwarzpurpurne bis dunkelrotbraune Blütenfärbung der am weitesten verbreiteten Sippe. Darauf nimmt auch der wissenschaftliche Artname Nigritella nigra (lat. nigritus $=$ schwärzlich, geschwärzt) Bezug.

\section{Verbreitung und Standort}

Kohlröschen sind auf europäische Hochgebirge beschränkt und kommen vor allem im Alpenraum und in Skandinavien vor. In den Alpen gehören Kohlröschen mit Edelweiß, Enzian und Almrausch zu den volkstümlichsten Pflanzen, denn ihre Blüten fallen wegen des starken Vanilleduftes auf und sind deshalb sehr beliebt. Zwei bis drei Blütenstände reichen aus, um ein Zimmer mit ihrem Duft zu erfüllen. Kohlröschen wachsen in den Alpen bevorzugt auf ungedüngten, mageren, niederrasigen Almwiesen in Höhenlagen von 1000 bis 2700 m. Das in Skandinavien vorkommende Kohlröschen ist auch in tieferen Lagen um $300 \mathrm{~m}$ anzutreffen. Je nach Art, Unterart oder Varietät bevorzugen sie entweder kalkhaltigen Untergrund oder Urgestein.

Zudem sind Kohlröschen auch im Kantabrischen Gebirge in Spanien, in den Pyrenäen (Foelsche 1997a, b), im Zentralmassiv und Jura in Frankreich, im Zentral-Apennin in Italien (Rossi et al. 1987), auf dem Balkan, in den Karawanken und den Karpaten (Buttlen 1991), in Nord-Griechenland sowie auf dem Peloponnes heimisch (Gembard \& Gölz 2002, Teppner \& Klein 1994). Im Schwarzwald kamen Kohlröschen in $800 \mathrm{~m}$ Höhe vor, doch sind sie dort ausgestorben.

\section{Merkmale der Gattung}

Nigritella-Arten sind mehrjährige Gewächse, die den Winter mit Hilfe von unterirdischen Speicherorganen überdauern. Es handelt sich um handförmige Knollen mit 2-5 Abschnitten. Während der Blütezeit sind pro Pflanze zwei Knollen entwickelt. Aus der dunklen, älteren Knolle entwickelt sich die aktuell blühende Pflanze und für das Folgejahr steht bereits eine jüngere und hellere Knolle bereit. Letztere ist von Häuten der alten Knolle, aus der sie hervorgegangen ist, umgeben. Da pro Vegetationsperiode nur eine einzige Knolle neu gebildet wird, unterbleibt die bei vielen anderen Orchideen auftretende vegetative Vermehrung. Kohlröschen stehen deshalb einzeln, an manchen Stellen allerdings durchaus noch zahlreich. 

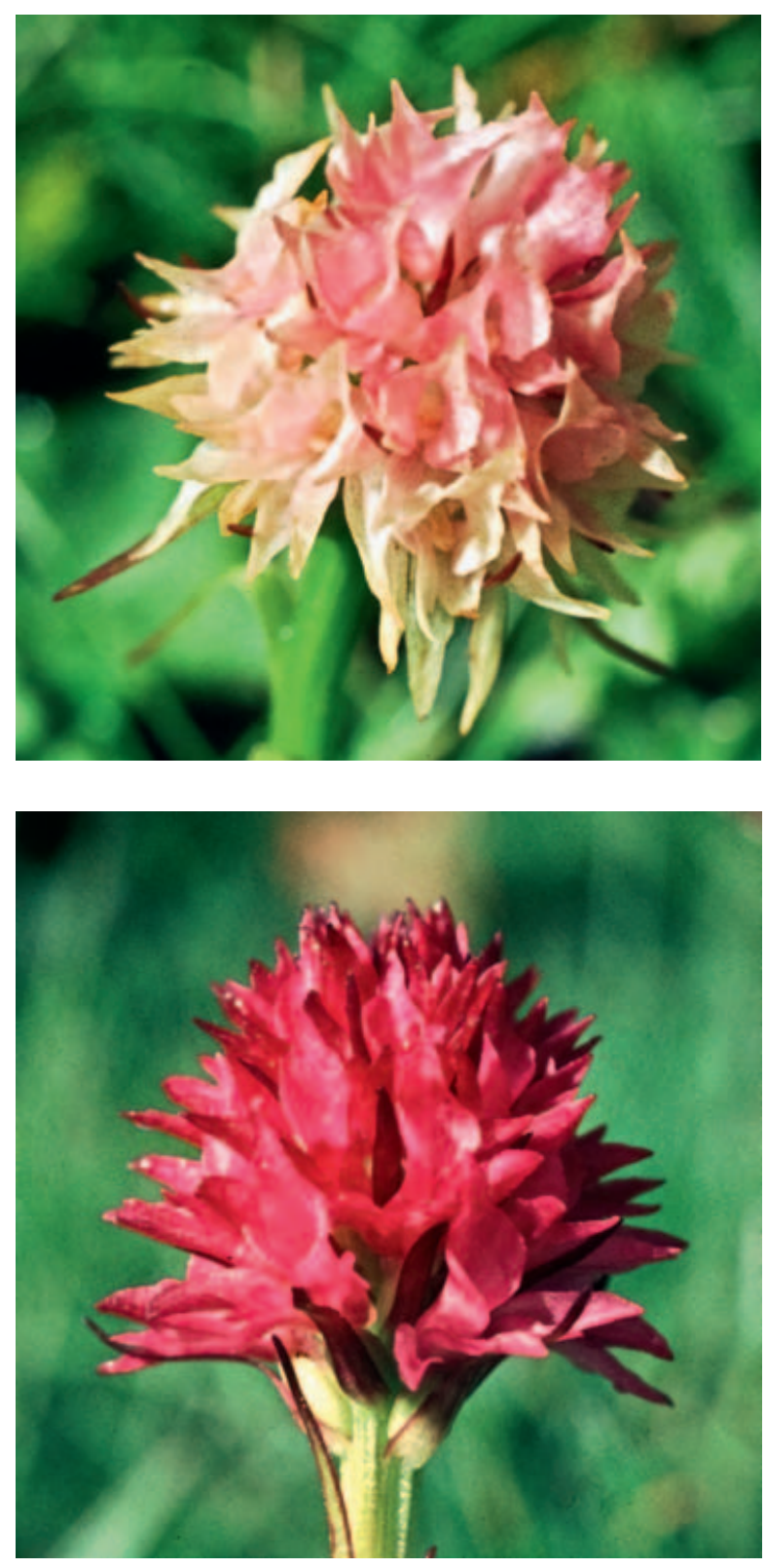

Mit einer Höhe von 5-25, seltener $35 \mathrm{~cm}$ sind Kohlröschen relativ niedrige Orchideen. Im Frühjahr treiben 10-12 grüne, ungefleckte und grasähnliche Blätter aus. Die meisten sind rosettig angeordnet und stehen aufrecht. Die Blütezeit liegt abhängig von Sippe (Art, Unterart oder Varietät) und Höhenlage des Wuchsortes zwischen Juni und August. Der dichte Blütenstand setzt sich aus 20-80 kleinen Blüten zusammen. Er ist je nach Sippe halbkugelig, kegelförmig oder zylindrisch geformt. Die Blüten stehen in den Achseln von spitzen, grünen, manchmal braunrot überlaufenen oder purpur gerandeten Tragblättern. Der Fruchtknoten ist im Gegensatz zu vielen anderen Orchideen nicht gedreht, sodass die Lippe, das größte der 6 Blütenblätter, schräg nach oben gerichtet ist. Sie kann somit nicht als Landeplatz für Bestäuber dienen. Die Lippe ist ungeteilt und ei- bis rautenförmig. Im unteren Drittel ist sie je nach Sippe mehr oder weniger sattelförmig eingeschnürt. Sie kann bis zu $12 \mathrm{~mm}$ lang werden, die übrigen Blütenblätter sind kürzer. Der sackartige, nektarhaltige Sporn ist mit 1-2 mm Länge sehr kurz. Das kurze Säulchen, an dem sich die Geschlechtsorgane befinden, wird nicht wie üblich von den sich helmartig zusammenneigenden Blütenblättern, sondern von der Lippe vor Regen geschützt.

\section{Vermehrung und Bestäubung bei Kohlrös- chen}

Im Gegensatz zu den hoch spezialisierten Ophrys-Arten, bei denen nur eine bestimmte Insekten-Art als Bestäuber fungiert, werden die Blütenstände des Kohlröschen von zahlreichen Insekten-Arten angeflogen. Schon 1874 wurden 48 Falter- und 5 andere Insekten-Arten als Blütenbesucher notiert. Genauere Überprüfungen zeigten später aber, dass die wenigsten Besucher Bestäuber sind. Denn an vielen Insekten wurden nach Besuch von Kohlröschen-Blüten keine Pollinien festgestellt. Zudem kam oft keine gerichtete Übertragung von Pollenpaketen zustande, da die Pollinien an den Füßen der Insekten hafteten und verloren gingen (BABORKA 1994). Nur wenige Tagfalter-Arten wurden mit Pollinien beobachtet, darunter Kleiner Fuchs (Aglais urticae) und Erebia-Arten (Mohrenfalter). Dagegen konnten mehrere tagaktive Nachtfalter-Arten mit Pollinien nachgewiesen werden, darunter diverse Blutströpfchen (Zygaena) sowie Grünwidderchen (Adscita statices), die auch zur Paarung oder als Ruheplatz rotfarbige Blüten anfliegen. Auch Eulenfalter wie z. B. KommaEule (Leucania comma), Graseule (Cerapteryx graminis) und die Baetische Tageule (Cleophana

Abb. 1 (oben): Die Farbe der Kohlröschen variiert zwischen Rosa und fast Schwarz; hier ein hellrosa Exemplar.

Abb. 2 (unten): Rotes Kohlröschen. 
baetica) kommen als Bestäuber von Kohlröschen in Betracht. Aus der Familie der Spanner wird nur Cleogene lutaris genannt (Vӧтн 2000). Unter den Kleinschmetterlingen sind die Zünsler Crambus perlellus und Diasemia literata Überträger von Nigritella-Pollinien. Die Besuche anderer Insekten, über die immer wieder berichtet wird, sind eher Zufallsbesuche und kaum mit einer gezielten Bestäubung in Verbindung zu bringen. Im Idealfall stammt der durch die Bestäuber übertragene Pollen von einer anderen Pflanze mit unterschiedlichem Erbgut (Fremdbestäubung, Allogamie). Dieser kann aber auch von der Nachbarblüte des gleichen Blütenstandes herrühren (Geitonogamie, von griech . geiton $=$ Nachbar) . Aufgrund des dichten Blütenstandes der Kohlröschen kommt es vermutlich häufig zu Geitonogamie und damit verbunden nur einem eingeschränkten Austausch von Erbgut. Eine Selbstbestäubung, d. h. die Übertragung der Pollenpakete auf die Narbe ein und derselben Blüte, ist bei den Kohlröschen nicht möglich, da die Pollinien unter den Narben liegen. Desweiteren trittbei bestimmten Kohlröschen-Sippen, die mehr als zwei Chromosomensätze haben, Apomixis auf. Dies ist eine Form der Fortpflanzung, bei der weder eine Reduktionsteilung noch eine Verschmelzung von Keimzellen stattfindet. Embryonen bzw. Samen bilden sich ohne Befruchtung. Bei Nigritella entwickelt sich der Embryo nicht aus einer unbefruchteten Eizelle (Jungfernzeugung, Parthenogenese), sondern aus einer vegetativen Zelle der Samenanlage. Die Nachkommen sind mit der Mutterpflanze genetisch identisch. Der Vorteil besteht darin, dass sie nicht auf Insektenbesuch angewiesen ist und selbst in für Insekten ungünstigen Witterungsperioden meist einen hundertprozentigen Fruchtansatz zeigt. Nachteilig ist, dass die Nachkommen aufgrund des gleichen Erbguts nur eine geringe Variabilität und damit Anpassungsfähigkeit aufweisen.

Abb. 3 (oben): Schwarzes Kohlröschen.

Abb: 4 (unten): Almwiese mit Kohlröschen.
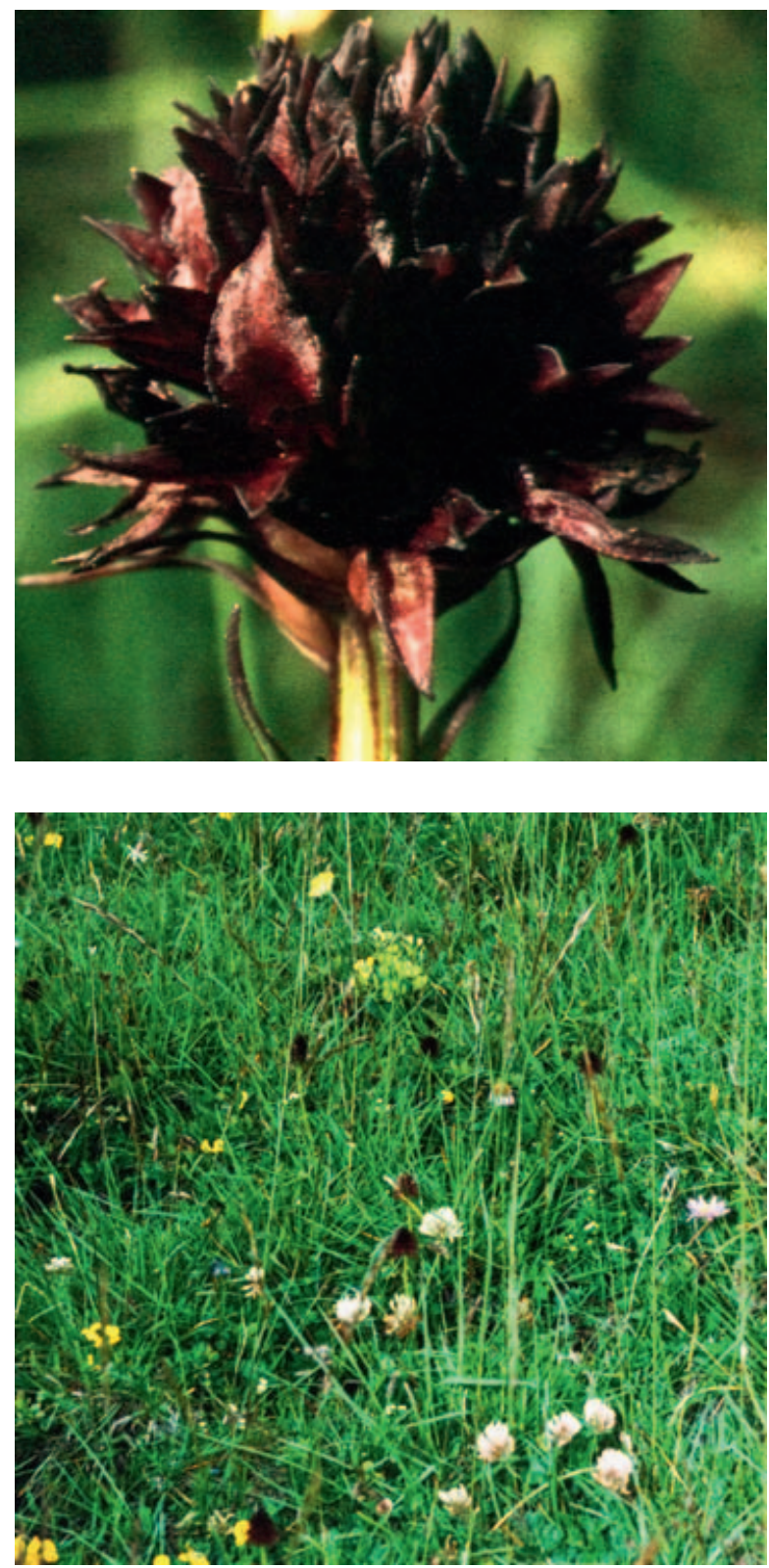

\section{Vielfalt der Kohlröschen}

Durch Störungen bei der Reduktionsteilung mit oder ohne nachfolgender Befruchtung entstehen Individuen mit verändertem Chromosomensatz. Eine Reihe von Kohlröschen-Sippen hat mehr als die üblichen zwei Chromosomensätze mit insgesamt 20 Chromosomen. Es kommen Chromosomenzahlen von 60, 80 und 100 vor, was einem drei-, vier- und fünffachen Chromosomensatz entspricht. In diesen Pflanzen kommt es während der Bildung von Geschlechtszellen zu Schwierigkeiten. Kohlröschen unterschiedlicher Chromosomenzahl unterscheiden sich auch (zumindest geringfügig) in ihrem äußeren Erscheinungsbild, ihren Arealen oder 

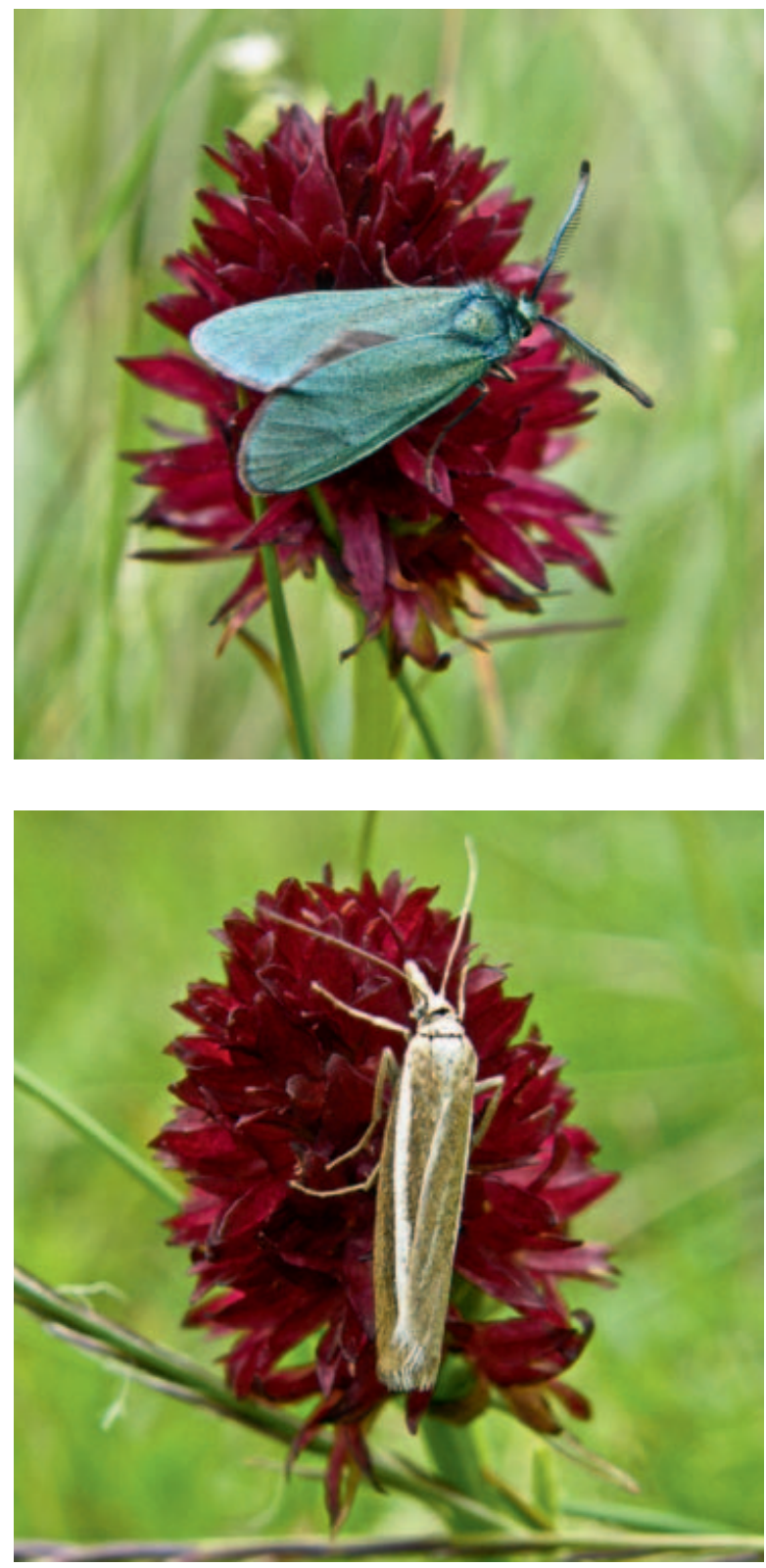

Standortsansprüchen. Nigritella wird deshalb in bis jetzt 18 Sippen untergliedert, während man in den 1950er Jahren nur zwischen dem Schwarzen (N. nigra) und dem Roten (N. rubra) Kohlröschen unterschied. Man nimmt heute an, dass fast jeder Gebirgszug, in dem Nigritella vorkommt, eine eigene Kohlröschen-Sippe vorweisen kann. Für die Unterscheidung der Sippen im Gelände gibt es Hilfen, die Bestimmung scheint aber nicht problemlos zu sein, weshalb es zu Streitfällen und unterschiedlichen Auffassungen über Einstufung und Verbreitung kommt. Dies gilt insbesondere für die westalpinen Kohlröschen, für die es keine Einigkeit bezüglich der Zuordnung gibt, offenbar weil karyologische Untersuchungen fehlen. Vermutlich gibt es weitere, noch nicht beschriebene Sippen wie z. B. ein Kohlröschen mit dem Arbeitsnamen $N$. macedonica aus Nord-Griechenland.

5.2 Nigritella-Sippen (nach Mrvicka I992, Almers et al. 1996, BrÜTSCH 2000, LÜNSMANN 2002, BAUMANn et al. 2006)

- archiducis-ioannis Teppner \& KLeIN 1985: benannt nach JoHANN, Erzherzog von Österreich (1782-1859); als Unterart von N. rubra aufzufassen; sehr selten im südöstlichen (steirischen) Salzkammergut (Totes Gebirge, Eisenerzer Alpen) auf Kalkmagerrasen. 2n = 80 .

- austriaca Teppner \& Klein I990: benannt nach Österreich aufgrund der Hauptverbreitung in den Ostalpen; von vielen als Unterart der skandinavischen Art $N$. nigra eingestuft; im französischen Raum wird sie als Art angesehen; nur auf basischen alpinen Magerrasen (Kalk, Dolomit); in den Ostalpen (Kärnten bis Niederösterreich), Dolomiten und in Kärnten. $2 \mathrm{n}=80$.

- bourneriasii E. \& R. BReiner I993: benannt nach Marcel Bournerias, Botaniker und Pflanzengeograf; die Sippe wurde von den Beschreibern als Unterart von $N$. corneliana eingestuft, wird aber wegen der Übergänge meist nur als Varietät angesehen; in den französischen und italienischen Westalpen. 2n $=40$.

- buschmanniae Teppner \& STER I996: benannt nach Dr. Adolfine Buschmann (19081989), tätig am Institut für Botanik der Universität in Graz; wird als Unterart zu N. rubra gestellt; verbreitet in den Südalpen, nur im nördlichen Teil der Brenta-Gruppe der Dolomiten (Italien), sehr selten auf kalkhaltigem Magerrasen in 1800-2500 m Höhe. $2 \mathrm{n}=100$.

- carpatica Zapal. 1906: Der Name leitet sich vom Vorkommen in den Karpaten ab; entweder als Varietät beschrieben oder als Art eingestuft und heute meist als Unterart von $N$. nigra

Abb. 5 (oben): Kohlröschen mit einem Grünwidderchen (Adscita spec.).

Abb. 6 (unten): Kohlröschen mit einem Rüsselzünsler (Crambidae, cf. Agriphila). 
angesehen; auf montanen, subalpinen, kalkarmen (!) Magerrasen und Weiden in 1000$1800 \mathrm{~m}$ Höhe in den rumänisch - ukrainischen Ostkarpaten. $2 \mathrm{n}=40$.

- cenisia G. \& W. Foelsche \& M. \& O. Gerbaud I998: benannt nach dem Hauptfundort am Col du Mont Cenis in den Savoyer Alpen; wurde ursprünglich als Art eingestuft, heute als Synonym von $N$. rhellicani betrachtet; auf Kalk in den französischen und italienischen Südwestalpen oberhalb von $2000 \mathrm{~m}$ und möglicherweise auch in den Südtiroler Zentralalpen. $2 n=40$ ?

- corneliana BEAUv. 1926: der Name bezieht sich auf die Schweizerin Cornelia Rudio, die erstmals die Pflanze fand; die Sippe wurde als Unterart von $N$. nigra beschrieben, später auch als Unterart zu lithopolitanica gestellt oder als eigene Art angesehen; verbreitet auf Kalk in den französischen und italienischen West- und Seealpen ab 1800-2500 m. 2n = 40 .

- dolomitensis TePpNer \& KLeIn 1998: benannt nach dem Vorkommen in den Dolomiten; als eigene Art oder Varietät von N. rubra angesehen; es gibt nicht nur die Probleme der Einstufung dieser Sippe; schwarzrot blühende Dolomiten-Populationen stehen in ihren Merkmalen oft zwischen denen der Unterarten austriaca und rhellicani. $2 \mathrm{n}=80$.

- gabasiana Teppner \& Klein 1993: der Name bezieht sich auf den Ort Gabas im Vallée d'Ossau in den Zentral-Pyrenäen; Unterart von $N$. nigra; auf Kalk und Urgestein von 1100-2450 m im Kantabrischen Gebirge (Spanien) und in den Pyrenäen. 2n = 40.

- gallica E. \& R. Breiner 1993: benannt nach der lateinischen Bezeichnung für Frankreich; Unterart oder Varietät von $N$. nigra; im nördlichen Teil der Westalpen östlich einer Linie Genf-Grenoble. 2n = 40 .

- iberica Teppner \& Klein i993: benannt nach dem lateinischen Namen für Spanien aufgrund des Vorkommens im Norden der iberischen Halbinsel; Unterart von $N$. nigra oder

Abb. 7: Händelwurz (Gymnadenia conopsea), mit der Nigritella leicht hybridisiert.

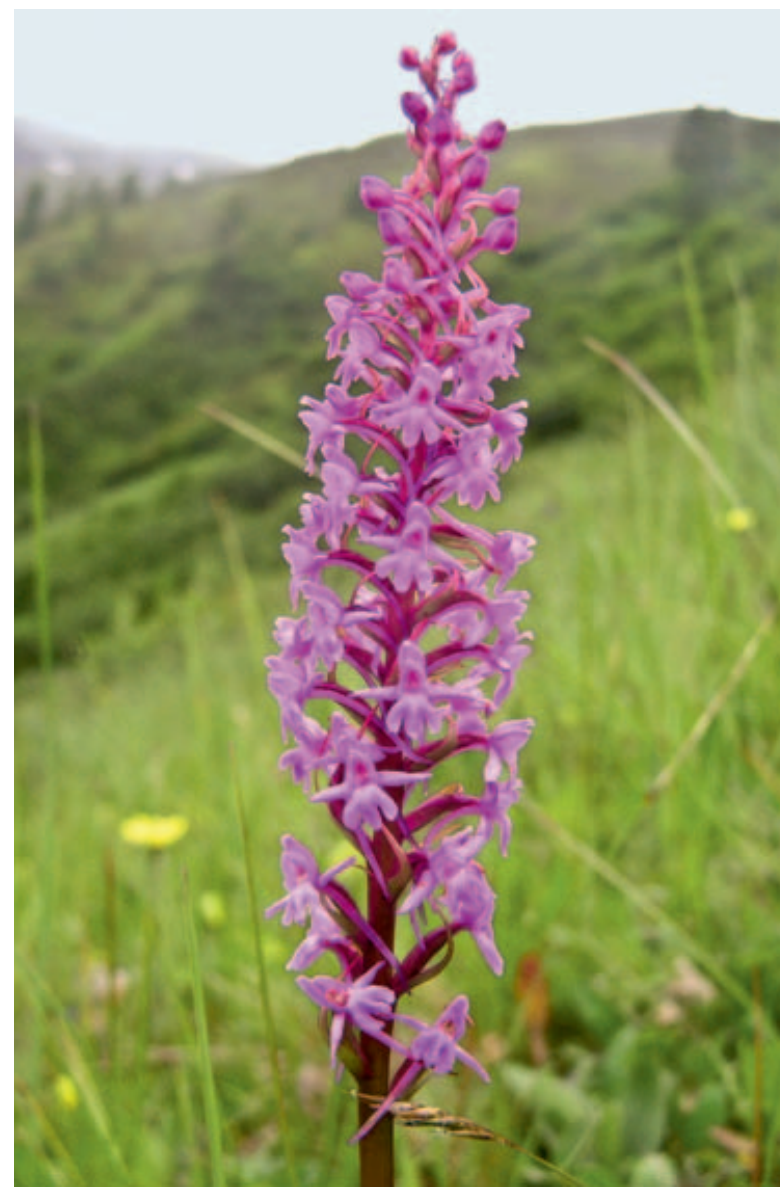

Varietät von austriaca; verbreitet im französischen Jura, in den französischen Westalpen, aber nicht in den Seealpen, im Zentralmassiv und in den Pyrenäen; weit verbreitet, aber nicht häufig; bevorzugt basisches Substrat und meidet sehr saure Böden. $2 \mathrm{n}=80$.

- lithopolitanica Ravnik 1978: der Name bezieht sich auf das Vorkommen in den Steiner Alpen nahe der Stadt Stein (slowenisch Kamniske Alpe, von gr. lithos = Stein und polis = Stadt); als Unterart von $N$. nigra eingestuft; auf Kalk von 1300-2100 m; selten in Slowenien und Österreich in den Südöstlichen Kalkalpen (Karawanken-Steiner- und Sanntaler Alpen, Koralpe, Lavanttaler Alpen). 2n = 40 .

- minor FoelsChe 2007: eine kleinere Form aus Kärnten.

- nigra L.: der Name bezieht sich auf die dunkle schwarzrote Blütenfarbe; lange Zeit als einzige Art der Gattung angesehen mit einer alpinskandinavischen Verbreitung; die reine Art sowohl auf kalkarmen Borstgraswiesen als auch in kalkreichen Silberwurzfluren von 300- 


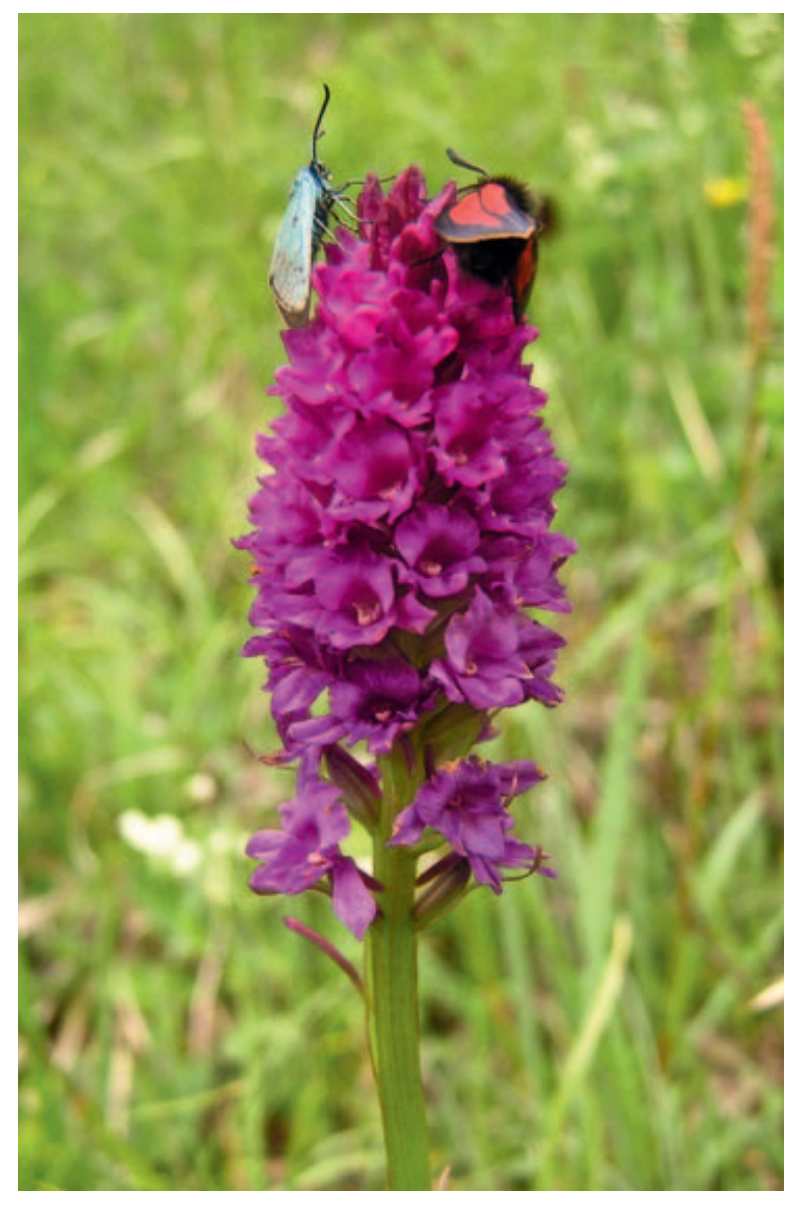

$1300 \mathrm{~m}$ in Jämtland (Mittelschweden und angrenzende norwegische Gebiete) und einem winzigen Areal in Nordnorwegen bei Tromsö. Mit dem Volksnamen Brunnella ist es die Landesblume von Jämtland. $2 \mathrm{n}=60$.

- rhellicani TeppNer \& Klein 1990: benannt nach dem Schweizer Johannes Müller, genannt RHELLICANUS. Er hatte in einem 1536 verfassten lateinischen Gedicht über eine Wanderung auf das Stockhorn als erster über ein Kohlröschen von schwarzbrauner Farbe, mit Moschusduft und handförmigen Wurzeln berichtet; Unterart von $N$. nigra; die in den Alpen am weitesten verbreitete und häufigste Sippe, fehlt nur in den äußersten östlichen Kalkalpen; außerdem kommt sie im Nord-Apennin, auf dem Balkan und in den Karpaten vor; sowohl auf Silikat als auch auf Kalk und Dolomit auf alpinen Magerrasen, extensiven Weiden und ungedüngten Mähwiesen. $2 \mathrm{n}=40$.

- rubra WeTtst. 1889: benannt nach der roten Blütenfarbe; meist als eigene Art betrachtet; nur auf Kalk bzw. Dolomit von 1200-2800 m in den nördlichen (Bayern) und südlichen Kalkalpen (vom Tessin bis Niederösterreich, Grazer Bergland), in den rumänischen Ostund Südkarpaten. Die rot blühende Sippe ist seltener als die dunkler gefärbten. $2 \mathrm{n}=80$.

- stiriaca K. Rechinger 1906: benannt nach den Vorkommen in der Steiermark; als eigene Art bzw. Varietät beschrieben oder als Unterart von N. rubra aufgefasst; selten in Oberösterreich, Salzburg (Dachstein) und der Steiermark (Sarstein bei Bad Aussee, Grazer Bergland); auf Kalk in 1350-2000 m Höhe. $2 \mathrm{n}=80$.

- widderi Teppner \& KLein 1985: benannt zu Ehren von Felix J. Widder (1892-1974), Universitätsprofessor für Botanik in Graz, der in Aufsammlungen von der Koralpe verschiedene Kohlröschen-Sippen erkannte, aber nicht publizierte; als Unterart von $N$. rubra eingeordnet; auf kalkhaltigen alpinen Magerrasen von 1300-2200 $\mathrm{m}$ in den nördlichen und südlichen Kalkalpen (Bayern, Tirol, Steiermark bis Niederösterreich, Grazer Bergland und Dolomiten) und im Zentral-Apennin (Abruzzen). 2n $=80$.

\section{Abgrenzung der Gattung}

Schon im 19. Jh. wurde bekannt, dass die Gattungen Nigritella, Händelwurz (Gymnadenia), Hohlzunge (Coeloglossum), Weißzüngel (Pseudorchis) und Knabenkraut (Dactylorhiza) nah verwandt sind. Deshalb hatte man schon früher vorgeschlagen, die Gattung Kohlröschen mit der Gattung Händelwurz zu vereinigen (Delforge I998), was aber wegen beträchtlicher morphologischer Unterschiede keineswegs allgemein akzeptiert wurde. Bei den Händelwurz-Arten ist beispielsweise die Lippe dreiteilig und nach unten gerichtet, bei den Kohlröschen einteilig und nach oben orientiert. Molekularbiologische Untersuchungen (BATEMAN et al. 1997, BATEMAN 2001) ergaben, dass Nigritella mit Gymnadenia zu vereinigen wäre. Nach anfänglicher Bereitschaft, der Auffassung zu folgen, hat sich

Abb. 8: Hybride zwischen Nigritella und Gymnadenia mit Grünwidderchen und Blutströpfchen (Zygaena). 
inzwischen wieder auch aufgrund molekulargenetischer Erkenntnisse an andereren Gen-Abschnitten die gegenteilige Meinung durchgesetzt (z. B. Wucherpfennig 2002). Kohlröschen hybridisieren gelegentlich mit verschiedenen Händelwurz-Arten ( $G$. conopsea, G. odoratissi$m a, G$. frivaldii), aber auch mit den Knabenkräutern Dactylorhiza fuchsii und D. majalis sowie mit dem Weißzüngel (Pseudorchis albida). Dies spricht für die enge Verwandtschaft der genannten Gattungen. Das Nicht-Drehen des Fruchtknotens wird dominant vererbt, so dass bei allen Bastarden die Lippe schräg bis aufrecht orientiert ist.

\section{Gefährdung}

Kohlröschen sind stellenweise durch Abpflücken für Blumensträuße, um den Duft ins Zimmer zu bringen, bedroht. Dies betrifft in der Regel aber nur einzelne Pflanzen. Eine weitaus größere Gefährdung liegt in der Vernichtung der Lebensräume. Durch Veränderung der Wirtschaftsweise kommt es zum Brachfallen der Almwiesen bzw. ihrer unzureichenden Beweidung. Dies wirkt sich auf die Nigritella-Populationen negativer aus als die möglichen Trittschäden des Weideviehs bei normaler Almwirtschaft; andererseits ist eine Überweidung natürlich auch schädlich. Besonders nachteilig sind die ungewollte Düngung aus der Luft und die beabsichtigte künstliche Düngung der Wiesen, die zur Nährstoffanreicherung führen und damit die Magerrasen zerstören. Die Lebensräume werden auch durch die Anlage von Straßen und Wegen, die die Höhenlagen für noch mehr Touristen erschließen. sowie durch den Bau von Skipisten und Skiliften bedroht. Nicht abzusehen sind Veränderungen, die als Folge der Erwärmung des Erdklimas auftreten werden. Es bleibt zu hoffen, dass die kleinen, aber feinen Kohlröschen auch in Zukunft noch in relativ großer Individuenzahl auf unseren Bergwiesen blühen und den Wanderer begeistern.

\section{Dank}

Für Anmerkungen zu den auf den Fotos abgebildeten Schmetterlingen danke ich Dr. WolfGANG NäSSIG.

\section{Literatur}

Almers, L., Newger, K. \& Wenker, D. 1996: Die Gattung Nigritella - ein allgemeiner Überblick sowie einige Funde in den Süd- und Ost-Alpen. Ber. AHO 13: 41-60.

BABORKa, M. 1994. Bestäuber von Nigritellen sowie Beschreibung eines Bastards zwischen Nigritella widderi und Gymnadenia. - Journ. Eur. Orch. 26: 88-93.

Bateman, R. 200I: Evolution and classification of European orchids, insights from molecular and morphological characters. - Jour. Eur. Orch. 33: 33-119.

Bateman, R., Pridgeon, A. \& Chase, M. i997: Phylogenetics of subtribe Orchidinae (Orchidoideae, Orchidaceae) based on nuclear ITS sequences. 2. Infrageneric relationships and reclassification to achieve monophyly of Orchis sensu stricto. - Lindleyana 12: 113-141.

Baumann, H. 2005: Nigritella Rich. - Die Orchideen Deutschlands. - In: Arbeitskreis heimischer Orchideen (Hrsg). S. 516-533. - Uhlstädt-Kirchhasel.

Baumann, H., Künkele, S. \& Lorenz, R. 1989:

Die nomenklatorischen Typen der von Linnaeus veröffentlichten Namen europäischer Orchideen. Mitt. Bl. AHO Bad.-Württ. 21: 35-700.

Beauverd, G. 1926: Le polymorphisme du Nigritella nigra (L.) Rchв.-Bull. Soc. Bot. Genève 17: 335-337. Breiner, E. \& R. 1989 : Ein neuer Fund von Nigritella archiducis-ioannis TePpNeR \& KLEIN. - Ber. AHO 6: 22-23.

Breiner, E. \& R. 1993: Beiträge zur Gattung Nigritella in den Westalpen. - Mitt. Bl. AHO Bad.-Württ. 25: 467-487.

BRÜTsCH, J.-P. 2000: Die Gattung Nigritella. -

Bauhinia 14: 21-32.

Buttler, K. P. i99i: Orchidaceae. - In: Strid, A. \& Tan, K.: Mountain flora of Greece, Bd. 2. - Edinburgh.

Delforge, P. 2003: La Nigritelle robuste du Mont Cenis (Savoie, France). - Natural. belges. 84: 117-132. Foelsche, G. \& W. I997a: Les Nigritelles des Pyrénées, de la Chaine cantabrique et du Massif central. L'Orchidophile 127: 111-116.

Foelsche, G. \& W. 1997: Les Nigritelles des Pyrénées, de la Chaine Cantabrique et du Massif Central. 2. Teil. L'Orchidophile 128: 152-58.

Foelsche, G., Foelsche, W. \& Gerbaud, W. I999: Nigritella cenisia Foelsche \& Gerbaud. - J. Eur. Orch. 31: 441-494, 487-494.

LÜnSManN, U. 2002: Die Gattung Nigritella - Eine Übersicht. - Jb. Naturwiss. Ver. Wuppertal 55: 303-307. Mrvicka, A. 1992: Die Arten der Gattung Nigritella in den Ostalpen. - Mitt. Bl. AHO Bad.-Württ. 24: 644-652.

RavniK, V. 1978: Beitrag zur Kenntnis der Gattung Nigritella Rich. IV. Nigritella lithopolitanica V. RavNIK, spec. nov. - Acta Bot. Croat. 37: 225-228.

Rossi, W., Capinieri, R., Teppner, H. \& Klein, E. I987: Nigritella widderi in the Apennines. - Phyton (Austria) 27: 129-138. 
Teppner, H. \& Klein, E. 1985: Karyologie und Fortpflanzungsmodus von Nigritella (Orchidaceae - Orchideae), inkl. $N$. archiducis-ioannis spec. nov. und zweier Neukombinationen. - Phyton 25: 147-176.

Teppner, H. \& Klein, E. 1990: Nigritella rhellicani spec. nov. und N. nigra (L.) Rchi. f. s. str. (Orchidaceae Orchideae). - Phyton 31: 5-26.

Teppner, Herwig \& Klein, Erich (1993): Nigritella gabasiana spec. nov., Nigritella nigra subsp. iberica spec. nov. (Orchidaceae - Orchideae) und deren Embryologie. - Phyton 33: 179-209.
Teppner, H. \& Klein, E. 1998: Etiam atque etiam: Nigritella versus Gymnadenia. Neukombinationen und Gymnadenia dolomitensis spec. nov. (Orchidaceae Orchideae). - Phyton 38: 220-224.

Teppner, H. \& Ster, T. 1996: Nigritella buschmanniae spec. nov. (Orchidaceae-Orchideae) und eine Biographie für Frau Adolfine Buschmann. - Phyton 36: 277-294. Vӧтн, W. 2000: Gymnadenia, Nigritella und ihre Bestäuber. - J. Eur. Orch. 32: 547-573.

Wucherpfennig, W. 2002: Nigritella: Gattung oder Untergattung. - Jahrb. Naturwiss. Ver. Wuppertal 55: $46-61$.

\section{Gärtnerisch-botanische Literatur}

Jan de Koning, Gerda van Uffelen, Alicja Zemanek \& Bogdan ZemaneK

Drawn after Nature. The complete botanical watercolours of the 16th-century Libri Picturati.

KNNV Publishing, Utrecht, 368 S., 24,8 x 34 cm, farbig illustriert mit über 1400 Skizzen und Abbildungen; ISBN 789050112383; 69,95 Euro.

Dieses voluminöse Werk umfasst Pflanzenaquarelle der Libri Picturati A18-30. Es handelt sich um eine Sammlung von mehr als 1400 Aquarellen mit Pflanzendarstellungen, die in der 2. Hälfte des 16. Jh. in den Niederlanden entstanden sind. Lange Zeit ruhten die Libri Picturati in Berlin, wo sie im 2. Weltkrieg verloren gingen. In den 1970er Jahren wurde diese einzigartige und aus kunsthistorischer Sicht extrem wertvolle Sammlung in Krakau wiederentdeckt. Seitdem erweckt sie bei Botanikern und (Kunst)historikern großes Interesse; es sind bereits zahlreiche wissenschaftliche Publikationen über diese Sammlung erschienen. Einem internationalen Expertenteam aus Leiden, Krakau, Madrid, Berlin und Rom ist es nun gelungen, die wunderschönen Pflanzenabbildungen komplett zusammen mit Kommentaren und historischen Anmerkungen in einem Buch zu veröffentlichen. In verschiedenen Aufsätzen wird versucht, die nicht ganz klare Entstehungs-Geschichte der Libri Picturati darzustellen. Dazwischen begeistern besonders die großformatigen, ganzseitigen und sehr naturgetreuen Darstellungen von Pflanzen.
Die Autorenschaft der Libri Picturati wird noch diskutiert. Vermutlich waren der berühmte Clusius und CHARLES DE SAINT OMER maßgeblich an der Entstehung der Bücher beteiligt. Beiden und ihren Arbeiten an den Libri Picturati widmet sich ausführlich der begleitende Text. Zudem werden technische Angaben zu Material und Verarbeitung der Bücher gegeben. Außerdem erfährt der Leser viel über die Geschichte botanischer Illustration und den botanischen Garten Leiden, aus dem die Pflanzenvorlagen stammen.

Im zweiten Teil des Buches sind alle Pflanzenabbildungen aus den Libri Picturati in numerischer Reihenfolge zusammen mit den Originalkommentaren abgedruckt. Leider sind die meisten Abbildungen etwas klein; eine größere Darstellung hätte allerdings den Rahmen des auch so schon recht dicken und schweren Buches gesprengt. Neben Wildund Zierpflanzen sind auch Nahrungs- und Heilpflanzen abgebildet.

Vermutlich wird man das Buch nicht von vorne bis hinten lesen, zumal die Aufsätze oft recht speziell sind. Es liefert aber viele spannende Details zu botanisch-kulturhistorischen Themen und ist eine Quelle für zahlreiche schöne historische Abbildungen von in Mitteleuropa heimischen oder dort bereits im 16. Jh. kultivierten Pflanzen. Es bereitet Botanikern und vor allem Freunden alter Pflanzendarstellungen sicherlich viel Freude. Für das qualitativ hochwertig verarbeitete Buch ist sein Preis recht moderat.

Hilke Steinecke 